\title{
Study of Coronagraphic Techniques
}

\author{
Volker Tolls ${ }^{1}$, Michael Aziz ${ }^{2}$, Robert A. Gonsalves ${ }^{3}$, \\ Sylvain Korzennik ${ }^{2}$, Antoine Labeyrie ${ }^{4}$, Richard Lyon ${ }^{5}$, \\ Gary Melnick ${ }^{1}$, Steve Somerstein ${ }^{6}$, Gopal Vasudevan ${ }^{6}$, \\ and Robert Woodruff ${ }^{7}$
}

\author{
${ }^{1}$ Harvard-Smithsonian Center for Astrophysics, 60 Garden St., Cambridge, MA 02138, USA \\ email: vtolls@cfa.harvard.edu \\ ${ }^{2}$ Harvard University, Cambridge, MA 02138, USA ${ }^{3}$ Tufts University, 161 College Ave., \\ Medford, MA $02155{ }^{4}$ Observatoire de Haute-Provence, 04870 Saint-Michel l'Observatoire, \\ France ${ }^{5}$ NASA/Goddard Space Flight Center, Code 935, Greenbelt, MD 20771 \\ ${ }^{6}$ Lockheed-Martin Corporation, 3251 Hanover Street, Palo Alto, CA94304 ${ }^{7}$ Lockheed-Martin \\ Corporation, P.O. Box 179, Denver, CO 80201
}

\begin{abstract}
SAO has set up a testbed to study coronagraphic techniques, starting with Labeyrie's multi-step speckle reduction technique. This technique expands the general concept of a coronagraph by incorporating a speckle corrector (phase and/or amplitude) in combination with a second occulter for speckle light suppression. The correction function is derived applying the phase diversity method on images taken in focus and slightly out-of-focus. The occulter masks for the testbed will initially be produced lithographically. However, in a parallel program we are studying a new manufacturing method. This method utilizes focussed ion beams and will directly mill the mask shape into absorbing material deposited on a transparent substrate.
\end{abstract}

Keywords. instrumentation: adaptive optics, instrumentation: miscellaneous, methods: laboratory, techniques: miscellaneous, (stars:) planetary systems.

\section{Introduction}

Direct imaging of extra-solar planets is important for determining the properties of individual planets and to study multi-planet systems. Obtaining spectra of extra-solar planets enables us to constrain the composition of planetary atmospheres and surfaces, their climates, and their rotation periods. The techniques required to isolate and detect an extra-solar planet next to its host star are quite challenging and require significant improvement. The dominant program for imaging exo-planets is NASA Terrestrial Planet Finder (TPF). The current requirements for the TPF telescope system are very challenging in regards to manufacturing the primary mirror and then in controlling the telescope environment such that the changes are extremely small. Further challenging requirements are set for the star light suppression system which basically tries to correct the wavefront of the light coming from an observed host star to near perfection before it enters the coronagraph. In our ongoing program, we are studying an alternate approach, Labeyrie's multi-stage speckle reduction (MSSR) technique, Labeyrie (2002), which should lower the requirements on the telescope system and possibly on its metrology as well. In $\S 2$ we describe the MSSR technique and present simulations showing its performance. In order to demonstrate the MSSR technique in the lab, we have designed a testbed which is described in $\S 3$. Essential elements of the testbed are soft-edge occulter masks. These masks are typically manufactured using lithographic methods, e.g. optical lithographic methods and High Energy Beam Sensitive (HEBS) glass photomasks. Our program is 


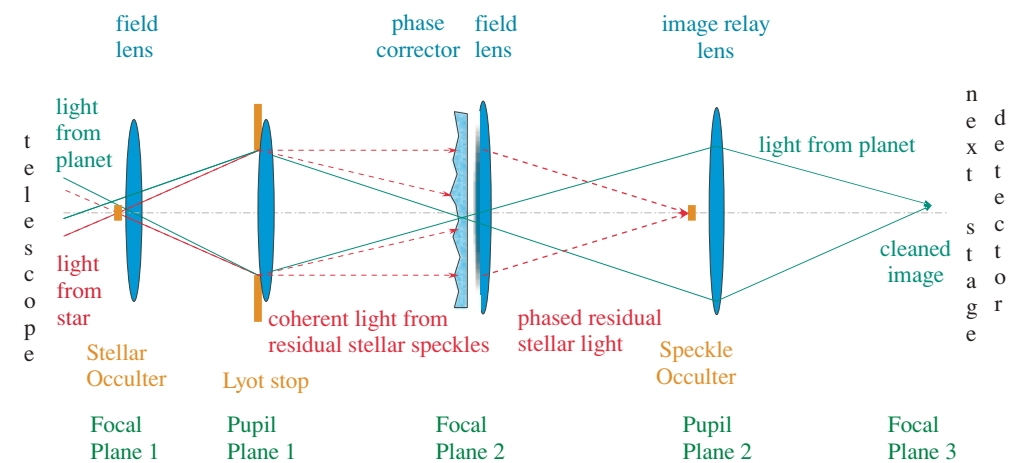

Figure 1. : Schematic setup for Labeyrie's multi-step speckle correction approach. The first part of this schematic shows a standard Lyot coronagraph (Focal Plane 1 and Pupil Plane 1) and a single correction step (phase corrector placed near Focal Plane 2, a second occulter near Pupil Plane 2), and a detection camera.

pursuing an alternate method utilizing an focused ion beam system to directly mill masks $(\S 4)$.

\section{Labeyrie's Multi-Step Speckle Correction Method}

\subsection{Background}

An alternative approach has been suggested by Labeyrie. Figure 1 shows an optical layout of Labeyrie's MSSR approach. Instead of correcting the telescope wavefront before the coronagraph, Labeyrie suggests that measurements be made of "the phase of the speckle pattern" in the image plane of a Lyot coronagraph. The speckle pattern is the result of phase errors on the primary mirror and other optics in the system up to the coronagraph. An adaptive element (e.g., a deformable mirror) is then applied with a conjugate of the phase in the speckle pattern, setting the net phase to approximately zero. This results in the speckle having a plane wave component emanating from the coronagraph focal plane. A lens following the corrector re-images the telescope pupil. The plane wave of scattered light will be mostly focused into a small spot in the center of the re-imaged pupil plane. A second occulter blocks the central spot and the result is re-imaged to another focal plane. Measurement of the phase must be made with a field that does not contain a planet or any other faint object (e.g. by imaging another star). This correction will then improve the contrast between the faint object and the speckle background by as much as a factor of 10 . Several additional stages of correction continue to improve the contrast ratio. Measurement and correction of the speckle phase need only be as accurate as $1 / 4$ wave, greatly relaxing the requirements on the adaptive optics (AO) system. The pupil plane occulter can be fairly large (e.g. up to $10 \%$ of the pupil diameter) without attenuating much of the signal of the off-axis planet or source. Imperfect correction of the focal plane phase causes the spot to spread out but it is still blocked by the stop. The Labeyrie approach will relax substantially the requirement that the wavefront accuracy of the large optics be better than $10^{-6}$ wave. Initial numerical simulations showed good results with a wavefront error of $1 / 1000$ wave of the initial wavefront which has to be demonstrated in the proposed laboratory tests. 

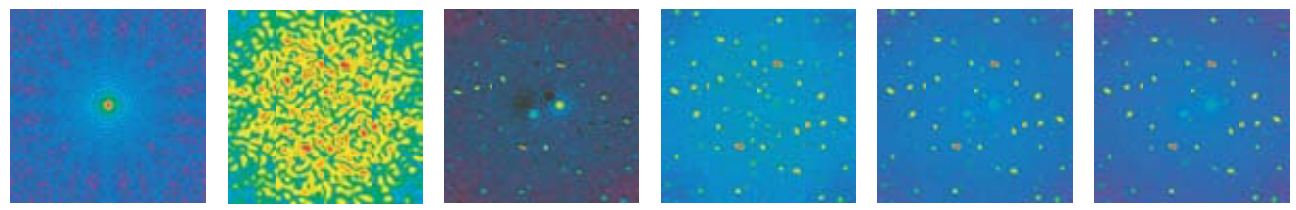

Figure 2. : Simulation of the Labeyrie multi-step method (for explanation see text).

\subsection{Simulations}

Before designing the testbed setup for the multi-step speckle correction technique, we simulated the approach using Fourier optics. The steps in our simulation are: (1) simulating the telescope, (2) simulating the coronagraph, (3) simulating the multistage speckle reduction, and (4) applying possible post-processing to the acquired images. The imperfections in the primary mirror are simulated using the specification of the power spectral distribution (PSD) for the TPF demonstration mirror. The resulting phase screen is then rescaled to the desired wavefront error. We assumed a wavefront error of $\lambda / 1000$; however, this number can be changed to any wavefront error. The occulter in the coronagraph can be selected. The current choices are a sinc ${ }^{2}$ or Gaussian profile absorbing mask. The mask parameters can be adjusted to optimize the system. Also, a rest-transmission can be specified to better simulate real occulters. We assumed a typical value of $10^{-10}$. The phase corrector did correct the phase on the same pixel raster as the simulation was performed. In our testbed, the phase corrector will not have such a high resolution.

Figure 2 shows a typical result of our simulations. The resolution is $512 \times 512$ pixels showing a FOV of $25 \lambda / \mathrm{D}$. The left panel shows the ideal point spread function and the second panel the speckle background after the star light has been suppressed by a soft-edge occulter and the exit pupil has been reduced to $90 \%$. The next three panels show the improvement of the speckle reduction after: 3 steps, 5 steps, and 7 steps. The simulated planets at $(4,0)^{*} \lambda / \mathrm{D}$ and at $(-2,-2)^{*} \lambda / \mathrm{D}$ can be identified after 5 to 7 steps. The right panel shows a post-measurement data processing step. The image from step 7 was rotated by 180 degrees and subtracted from the original image. The speckles show a very high, but not perfect, rotational symmetry. In addition, the image had to be shifted by 1 pixel vertically and horizontally for the best line-up of the speckles. The planets still show up as circular objects with a point-symmetric, negative counterpart. Since the shape of the planets seems to be invariant when shifting the image and the "shadows" of the speckles (the negative component) move around the speckles, planets should be easily identifiable. The number of speckle reduction steps can be reduced, if both, the phase and the amplitude, can be corrected. In this case, only 1 to 2 steps might be required assuming sufficient resolution in the correcting components.

\section{Testbed Design}

In the initial design, SAO's coronagraphic testbed consists of the following elements: 1) the simulated source, 2) the Lyot coronagraph, 3) a single speckle reduction step, and 4) the camera. Initially, we are using a $1 \mathrm{~mW}$ He-Ne laser. The output beam is fed into a spatial filter with a $30 \mathrm{~mm}$ objective and a $10 \mu \mathrm{m}$ pinhole. The transmitted light enters the coronagraph uncollimated. The coronagraph consists of a 2-inch diameter, spherical mirror with a surface accuracy of $\sim \lambda / 1000$ at $633 \mathrm{~nm}$ at a distance of about 2 meters from the pinhole. The now converging beam passes through an apodizer with a cosine function and focuses onto the occulting mask. The final elements of the coronagraph are the Lyot stop followed by a 1-inch, 0.5 -m radius spherical mirror.

The phase correcting element in our single step speckle corrector is a deformable mirror from Boston Micromachines Inc. This mirror has 140 elements, a 12-by-12 pixel array 

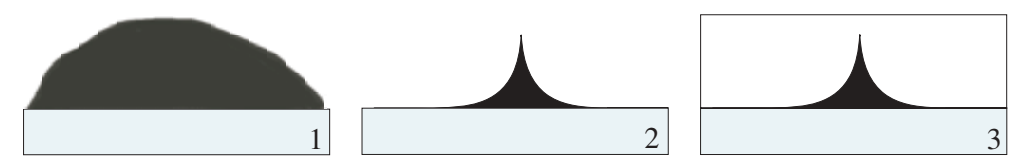

Figure 3. : Procedural steps in the manufacturing of ion beam milled occulter masks.

with the corner mirrors missing. The size of a single pixel is $275 \times 275 \mu \mathrm{m}(300 \mu \mathrm{m}$ spacing), the total array size is 3.3 by $3.3 \mathrm{~mm}$, and the stroke per element is $2 \mu \mathrm{m}$ with $2 \mathrm{~nm}$ repeatability. The corrected speckle light is focused on a second occulter, effectively eliminating most of the speckle light. The final "cleaned" image is focused onto the detector, a Apogee ST-401 CCD camera with a Kodak full-frame CCD KAF-0401E. The camera is mounted on a z-direction translation stage. This enables us to use phase diversity to retrieve the phase and amplitude of the wave (see Gonsalves \& Tolls 2005) and to calculate the correction function for the deformable mirror.

\section{Ion Beam Milling of Occulter Masks}

Our efforts include the study of a new method of mask manufacturing. The basic steps of this method are outlined in Figure 3. This effort uses the FEI Dual Beam Focused Ion Beam System (FIB) consisting of a fully digital Field Emission Scanning Electron Microscope integrated with Focused Ion Beam Technology at Harvard's Center for Imaging and Mesoscale Structures (CIMS). Currently we are studying how to control the ion beam in order to produce the desired shape. The build-in electron microscope enables us to tightly control the milling process. The challenge is to solve are finding the best milling patterns for removing the material without leaving marks on the mask or re-depositing removed material. In parallel we are studying how errors in the mask shape effect the mask properties and performance. Utilizing the beam propagation method for simulations, we can determine the mask behavior from theoretical shapes and wfrom measured shapes.

\section{Mask Tester}

For characterization of the manufactured occulter masks, we have designed a mask tester. The mask tester consists of a He-Ne laser source, collimating optics, $\mathrm{x}-$ and $\mathrm{y}$ translation stage, pinhole, detector, and control electronics (a schematic of the setup can be found in Tolls et al. (2005)). The laser beam is focused with a focal spot size of about $1 \mu \mathrm{m}$. The location of the focal spot will be in the mask plane. The occulter will be mounted on an xy-translation stage whichcan be moved with $50 \mathrm{~nm}$ repeatability over a 1-inch range. The transmitted beam is then fed through a pinhole onto a pin diode detector. The test setup will be computer controlled and needs only an initial rough alignment such that the mask is completely within the scan range. After scanning, the mask profile can be retrieved using the maximum entropy method.

\section{Acknowledgements}

This work was supported by NASA through grant number NNG04GC57G and Smithsonian Astrophysical Observatory IRAD funding.

\section{References}

Gonsalves, R.A. \& Tolls, V. Proc. SPIE 5905, in press

Labeyrie, A. Proc. ESLAB 36 symposium, ESTEC, ESA SP-514

Tolls, V. et al. Proc. SPIE 5905, in press

TPF Program Technology Demonstration Mirror, Detailed Equipment Specification, JPL 